\title{
Integrability for the Derivative Formulas of Rotation Minimizing Frame in Euclidean 3-Space and Its Applications
}

\author{
Firat Yerlikaya* and İsmail Aydemir \\ (Communicated by Yusuf Yayl1)
}

\begin{abstract}
We analyze integrability for the derivative formulas of the rotation minimizing frame in the Euclidean 3-space from a viewpoint of rotations around axes of the natural coordinate system. We give a theorem that presents only one component of the indirect solution of the rotation minimizing formulas. Using this theorem, we find a lemma which states the necessary condition for the indirect solution to be a steady solution. As an application of the lemma, the natural representation of the position vector field of a smooth curve whose the rotation minimizing vector field (or the Darboux vector field) makes a constant angle with a fixed straight line in space is obtained. Also, we realize that general helices using the position vector field consist of slant helices and Darboux helices in the sense of Bishop.
\end{abstract}

\section{Introduction}

\subsection{Short History of Curves}

The Frenet equations were originally introduced in 1831 by Karl Eduard Senff and Johann Martin Bartels, gaining simplicity and usefulness to the theory of space curves. In 1847, they were reintroduced in the dissertation of Jean Frederic Frenet, which was published in 1852. Soon after, those equations were found independently by Joseph Alfred Serret in 1851, and are sometimes called Frenet-Serret equations (for details on this early history, see [17]). The achievement of the Frenet approximation for a space curve was emerged around 1880 by the French mathematician, Jean Gaston Darboux, with the describing of the vector $w=\tau T+\kappa B$. The instantaneous change of the Frenet frame can be measured with a rotation around this vector. On the other hand, since the principal normal vector field always points to the center of curvature, one may see that there exists unnecessary-rotation in the frame. Although the Frenet frame is the most basic tool used to solve many problems in the field of biology, mechanics and engineering, etc., especially because of the unnecessary-rotation, it loses its function for many areas such as camera, fluid flow, visualization, robotic, integrable system and quantum mechanics. Therefore, the first thing that comes to mind is the question of whether there is a moving frame with the property of minimum bending. In [7], the admissible answer has been given by Richard Lawrence Bishop as "there is more than on way to frame a curve". It is generally called Bishop frame. He performed this by showing that parallel fields over a $C^{2}$ regular curve are formed a three-dimensional vector space over $\mathbb{R}$, and thus it is sometimes called a relatively parallel adapted frame. In recent years, looking at basic literature on this frame, the readers will observe another name due to the 
property of the minimum bending, which is called rotation-minimizing frame (briefly RMF).

\subsection{Literature Review on Position Vector Field of a Curve}

There exist two main methods to determine the position vector field of a smooth curve as follows:

- According to the moving frame

- According to the standard frame.

The first one is to create a system of ordinary differential equations by writing the smooth curve to be linear in terms of moving frame vectors with differentiable function coefficients. The second one is to convert the derivative formulas consisting of three vector differential equations into a single vector differential equation. Surveys on them are listed below:

In 2010, Ahmed Tawfiq Ali and his coauthor investigated the problem of determining the position vector field of a smooth curve satisfying a geometric property that the principal normal vector field makes a constant angle with a fixed straight line, which is called slant helix. They found a vector differential equation of third order that accepts the principal normal vector field as the dependent variable. Thus, the position vector field of a timelike slant helix with respect to standart frame of Minkowski space was obtained according to natural equations such that the straight line is parallel to $e_{3}$, which is a special solution of this vector differential equation [1]. For slant helices in the Euclidean 3-space, see [2].

In 2011, similar work was done to find the position vector field of a general helix with respect to both Frenet frame and standart frame in Euclidean 3-space [3]. For timelike and spacelike general helices in Minkowski 3-space, respectively, see [4, 5].

In 2012, Ali found the position vector field of an arbitrary curve with respect to both methods in Galilean 3-space. As an application, he obtained that of some special curves such as straight line, plane curve, circular helix, general helix, Salkowski curve and anti-Salkowski curve in Galilean 3-space [6]

In 2014, the authors found the position vector field of a general helix in Galilean 3-space through a vector differential equation of fourth order [16].

In 2014, the authors proved that the position vector field of an arbitrary curve with respect to Frenet frame (i.e. moving frame) in Galilean 3-space satisfies a vector differential equation of second and fourth-order. Moreover, they found the position vector of a unit speed rectifying curve [18].

Except for the methods above, the position vector field of special curves such as helix and slant helix can also be obtained by using the associated curves and the helical surfaces. In [10], the authors described two new concepts from a Frenet curve in the 3-dimensional Euclidean space, which are called principal direction curve and principal donor curve, and they provided a method to construct general helices and slant helices through these curves. Subsequently, analogues of this problem are done in 3-dimensional Lorentz-Minkowski space $\mathbb{R}_{1}^{3}$, 3-dimensional Compact Lie group $\mathbb{G}$, anti-de Sitter 3-space $\mathbb{H}_{1}^{3}$ and three-and four-dimensional Galilean space $\mathbb{G}^{3,4}[11,12,14,15]$. Finally, in 2016, Pascual Lucas and Jose Antonio Ortega-Yagues presented two methods to create slant helices in helix surfaces [13].

\subsection{Motivation}

In the previous part, it is mentioned that the problem of determining a natural representation of the position vector field for a smooth curve with respect to the Frenet frame has been investigated by the aforementioned methods, and consequently, the position vector field of special curves such as general helices and slant helices has been computed in the natural representation form. The most key point of the methods used to find this representation is that the first two vector fields (denoting by $T$ and $N$ ) of the Frenet frame have a direct connection with each other. When it comes to the position vector field of a smooth curve wrt the rotation minimizing frame, it is clear that the presented methods are inadequate due to the lack of the connection which we referred to above. Therefore, our motivation is to present a new method that fills the scientific gap in the position vector field with respect to the rotation minimizing frame.

\section{Preliminaries}

When we equip the real vector space $\mathbb{R}^{3}$ with the standart flat metric (called the Euclidean metric, generally) given by:

$$
g=d x^{2}+d y^{2}+d z^{2}
$$


the resulting space called as the Euclidean space and denoted by $\mathbb{E}^{3}$, where $(x, y, z)$ is an usual coordinate system of $\mathbb{E}^{3}$. The norm of an arbitrary vector $w \in \mathbb{E}^{3}$ is defined as $\|w\|=\sqrt{g(w, w)}$. Also, for two non-zero vectors $u=\left(u_{1}, u_{2}, u_{3}\right)$ and $v=\left(v_{1}, v_{2}, v_{3}\right)$ in $\mathbb{E}^{3}$, recall that the cross product of $u$ and $v$ is defined as

$$
u \times v=\left|\begin{array}{ccc}
i & j & k \\
u_{1} & u_{2} & u_{3} \\
v_{1} & v_{2} & v_{3}
\end{array}\right| .
$$

Let $r: I \rightarrow \mathbb{E}^{3}$ be a smooth curve parametrized by the arbitrary parameter $t$, where $I$ is an open subset of $\mathbb{R}$. Its norm is given by $\|r\|=\sqrt{g(r, r)}$ and $r$ is called a unit speed curve parametrized by the arc lenght $s$ if its velocity vector $r^{\prime}$, i.e. the first derivative of the curve, satifies $\left\|r^{\prime}\right\|=1$. From now on, the parameter of $r$ will always be $s$. When denoting the Frenet-Serret frame along the curve $r$ by $\{T, N, B\}$ in the Euclidean 3-space, its derivative formula is given by

$$
\left(\begin{array}{c}
T^{\prime} \\
N^{\prime} \\
B^{\prime}
\end{array}\right)=\left(\begin{array}{ccc}
0 & \kappa & 0 \\
-\kappa & 0 & \tau \\
0 & -\tau & 0
\end{array}\right)\left(\begin{array}{l}
T \\
N \\
B
\end{array}\right),
$$

where $\kappa$ and $\tau$ are the curvature and the torsion functions of $r$, respectively.

On the other hand, the derivative formulas of the rotation minimizing frame denoted by $\left\{T, M_{1}, M_{2}\right\}$ along $r$ are below:

$$
\left(\begin{array}{c}
T^{\prime} \\
M_{1}^{\prime} \\
M_{2}^{\prime}
\end{array}\right)=\left(\begin{array}{ccc}
0 & k_{1} & k_{2} \\
-k_{1} & 0 & 0 \\
-k_{2} & 0 & 0
\end{array}\right)\left(\begin{array}{c}
T \\
M_{1} \\
M_{2}
\end{array}\right),
$$

where $k_{1}$ and $k_{2}$ are the rotation minimizing curvature functions of $r$ and $M_{1}, M_{2}$ are arbitrary unit vector fields in $\mathbb{E}^{3}$. The geometric apparatus between the rotation minimizing frame and the Frenet-Serret frame, which we referred to before, are given by

$$
\begin{gathered}
\left(\begin{array}{c}
T \\
N \\
B
\end{array}\right)=\left(\begin{array}{ccc}
1 & 0 & 0 \\
0 & \cos \mu(s) & -\sin \mu(s) \\
0 & \sin \mu(s) & \cos \mu(s)
\end{array}\right)\left(\begin{array}{c}
T \\
M_{1} \\
M_{2}
\end{array}\right), \\
\kappa=\sqrt{k_{1}^{2}+k_{2}^{2}} \\
\tau=\mu^{\prime}(s)
\end{gathered}
$$

where $\mu(s)=\arctan \left(\frac{k_{1}}{k_{2}}\right)$ [7]. By Eq. (2.2) and the angle $\mu$, we have the following theorem:

Theorem 2.1. Let $r=r(s)$ be a smooth curve with rotation minimizing curvatures $k_{1} \neq 0$ and $k_{2} \neq 0$. Then, $r$ is a general helix if and only if the rotation minimizing curvatures of the curve satisfy

$$
\frac{k_{1}^{2}}{\left(k_{1}^{2}+k_{2}^{2}\right)^{\frac{3}{2}}}\left(\frac{k_{2}}{k_{1}}\right)^{\prime}=\text { constant }
$$

\section{Integrability for the rotation minimizing formulas}

The problem of finding two functions of one parameter (obviously, the curvatures $k_{1}$ and $k_{2}$ ) of a given smooth curve is investigated in differential geometry courses at undergraduate level. The reverse problem is, however, at graduate level, and it is called as the determination of a natural representation of the position vector field for a smooth curve or known as solving natural (intrinsic) equations. For the rotation minimizing frame $\left\{T, M_{1}, M_{2}\right\}$, one may regard Eq. (2.1) as the following form:

$$
\left\{\begin{aligned}
\frac{d T}{d s} & =f\left(s, T, M_{1}, M_{2}\right) \\
\frac{d M_{1}}{d s} & =g\left(s, T, M_{1}, M_{2}\right) \\
\frac{d M_{2}}{d s} & =h\left(s, T, M_{1}, M_{2}\right),
\end{aligned}\right.
$$


where the functions $f, g$ and $h$ are considered to be linear functions with coefficients that depend on $s$. Examining the above system, the readers see a system of vector differential equations in which $T, M_{1}$ and $M_{2}$ are dependent variables. As it is known, the most important fact in the theory of differential equations is to ensure the existence and uniqueness of their solutions. In the Euclidean 3-space, the existence and uniqueness of solutions for Frenet formulas in the fundamental theorem of the local theory of curves have been explicitly expressed and proved in Manfredo Do Carmo's book published in 1976, considering a system of nine differential equations (for details, the readers can look at the book named Differential Geometry of Curves and Surfaces) [9]. On the other hand, despite the fact that the existence and uniqueness of the solution curve for each differentiable curvature functions are guaranteed, solutions can be long, complicated and in general not obtained by integration, i.e may not be steady. In relation to that, as mentioned in the introduction section, the general case is still an open problem, and there are only solutions for a few types of special curves, in fact, no solution could be developed in the rotation minimizing frame. Naturally, it appears the following question supporting the motivation of our work:

Question 1. Is it possible to present a new method in such a way that the derivative formulas of the rotation minimizing frame in the Euclidean 3-space $\mathbb{E}^{3}$ can easily be integrated?

Let $\mathbb{E}^{3}$ endow the Euclidean 3-space. It is well-known that a natural orthonormal basis of $\mathbb{E}^{3}$ that $B=$ $\left\{e_{1}=(1,0,0), e_{2}=(0,1,0), e_{3}=(0,0,1)\right\}$. We suppose the coordinates of a vector with respect to $B$ that $\{x, y, z\}$. We set up another ordered orthonormal basis $B^{\prime \prime}=\left\{e_{1}^{\prime \prime}, e_{2}^{\prime \prime}, e_{3}^{\prime \prime}\right\}$ and the new coordinate system $\left\{x^{\prime \prime}, y^{\prime \prime}, z^{\prime \prime}\right\}$ associated with it using the following rotations, respectively, by means of $\{x, y, z\}$

- around the $z$-axis

- around the $x^{\prime}$ axis

- around the $y^{\prime \prime}$ axis

such that

$$
\begin{aligned}
& e_{j}^{\prime \prime}=\frac{e_{1}^{\prime \prime} \times e_{i}}{\left\|e_{1}^{\prime \prime} \times e_{i}\right\|}, \quad j=2,3, \quad i=1,2,3 \\
& \left(e_{1}^{\prime \prime} \times e_{j}^{\prime \prime}\right)=\left\{\begin{array}{ccc}
e_{3}^{\prime \prime} & , & j=2 \\
-e_{2}^{\prime \prime} & , & j=3 .
\end{array}\right.
\end{aligned}
$$

Note that $y^{\prime \prime}$ is obtained after first two rotations.

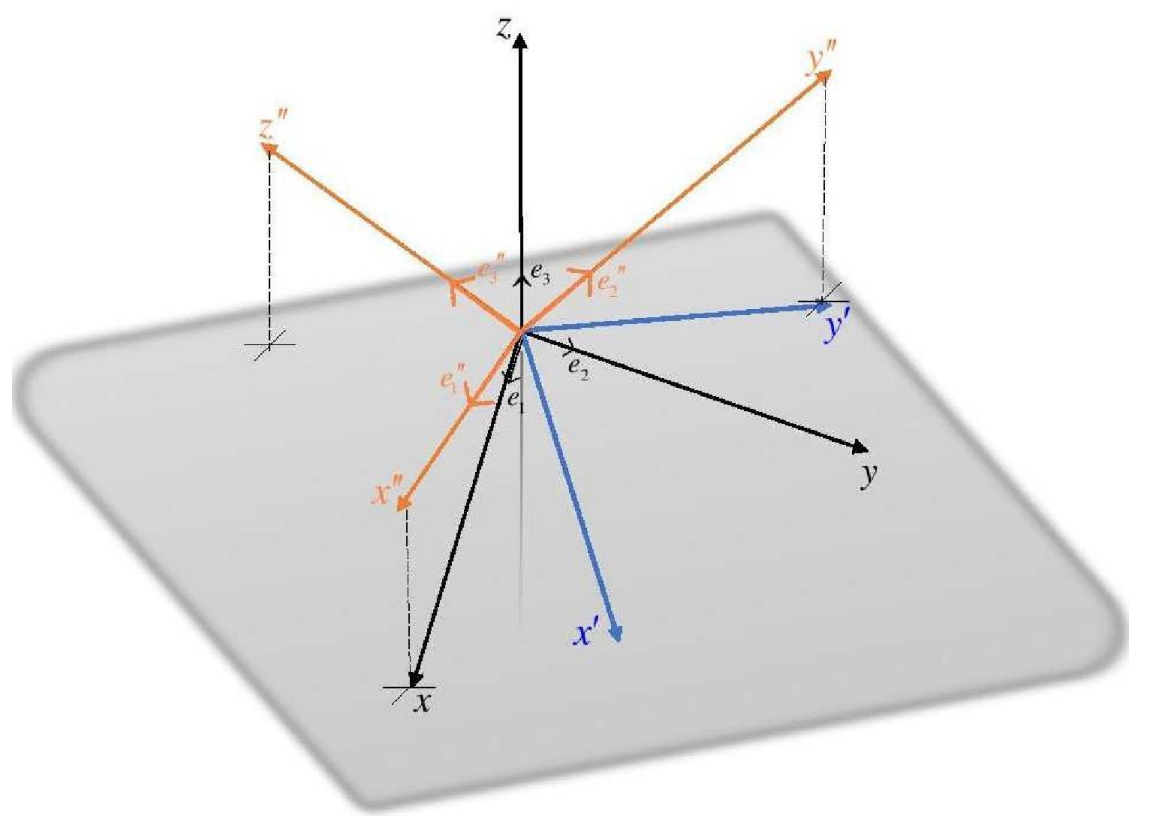

Figure 1. The new coordinate system associated with standard coordinate system. 
Let $r: I \rightarrow E^{3}$ be a smooth curve parametrized by the arc length $s$ such that $r^{\prime \prime}(s) \neq 0, s \in I$ and let $\left\{T, M_{1}, M_{2}, k_{1}, k_{2}\right\}$ be its RMF apparatus at the point $r(s)$. Now, assume that an arbitrary curve $\bar{r}$ derives from the curve $r$ by a rigid motion in such a way that the tangent vector field $\bar{T}$ at the point $\bar{r}\left(s_{0}\right)$ of $\bar{r}$ coincides with the vector field $e_{1}^{\prime \prime}$. As a result of this rigid motion, $\bar{M}_{1}$ and $\bar{M}_{2}$ lie in the plane spanned by $e_{2}^{\prime \prime}$ and $e_{3}^{\prime \prime}$. Note that the remaining vector fields of $\bar{r}$ denote by $\bar{M}_{1}$ and $\bar{M}_{2}$, respectively. Thereby, it is natural to talk about a transition matrix between the systems $\left\{\bar{T}, \bar{M}_{1}, \bar{M}_{2}\right\}$ and $\left\{e_{1}{ }^{\prime \prime}, e_{2}{ }^{\prime \prime}, e_{3}{ }^{\prime \prime}\right\}$, and its form is as follows:

$$
\left(\begin{array}{c}
\bar{T} \\
\bar{M}_{1} \\
\bar{M}_{2}
\end{array}\right)=\left(\begin{array}{ccc}
1 & 0 & 0 \\
0 & \cos \theta(s) & -\sin \theta(s) \\
0 & \sin \theta(s) & \cos \theta(s)
\end{array}\right)\left(\begin{array}{c}
e_{1}^{\prime \prime} \\
e_{2}^{\prime \prime} \\
e_{3}^{\prime \prime}
\end{array}\right)
$$

where $\theta$ is an angle between the vector fields $\bar{M}_{1}$ and $e_{2}^{\prime \prime}$.

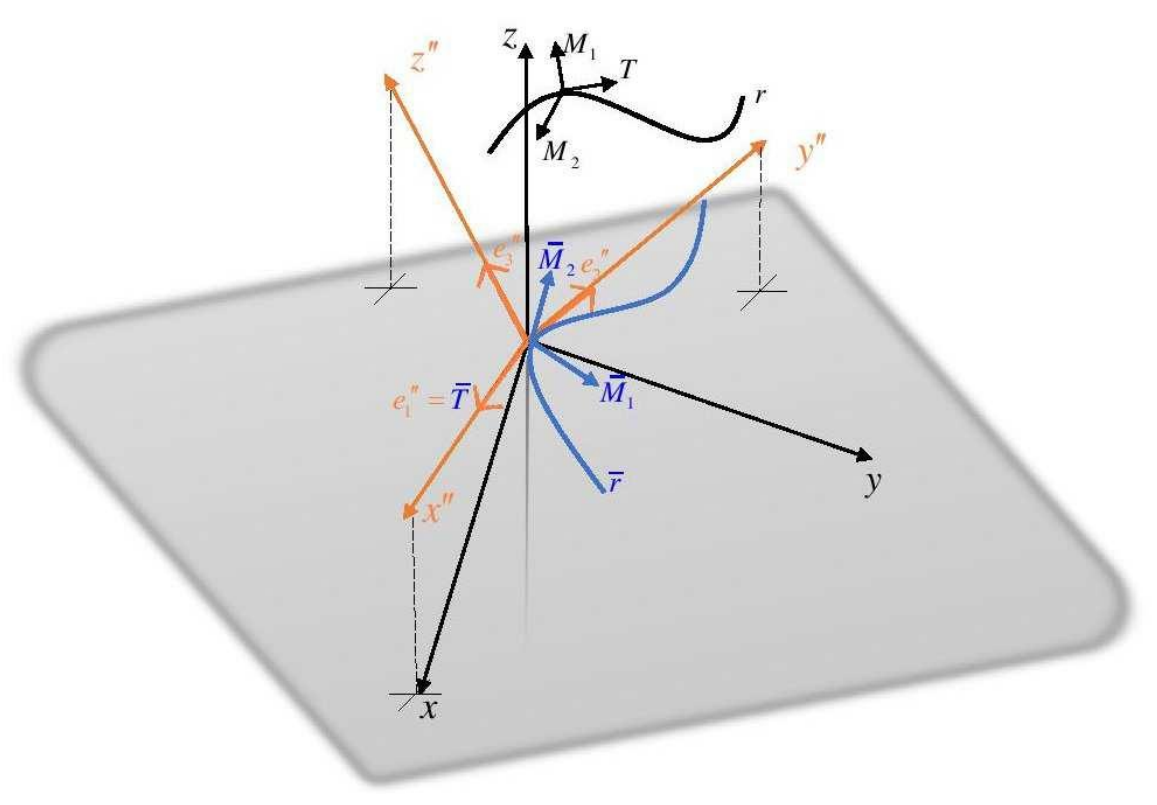

Figure 2. The location of the rotation minimizing vector fields of the desired curve in the new coodinate system.

On the other hand, it should be remarked that the rigid motion which takes $\bar{r}\left(s_{0}\right)$ into $r\left(s_{0}\right)$ and $\bar{T}, \bar{M}_{1}, \bar{M}_{2}$ into $T, M_{1}, M_{2}$ is actually same with the rigid motion reffered to above. Thus, we have

$$
T=\bar{T}, M_{1}=\bar{M}_{1}, M_{2}=\bar{M}_{2}
$$

for every points $s=s_{0}$.

Writing $i=1$ and $j=2$ by reasoning as $e_{1}^{\prime \prime}=\bar{T}=T=\left(T_{1}, T_{2}, T_{3}\right)$, we have the basis vectors of the new coordinate system as follows:

and

$$
e_{2}^{\prime \prime}=\frac{1}{\sqrt{1-T_{1}^{2}}}\left(0, T_{3},-T_{2}\right)
$$

$$
e_{3}^{\prime \prime}=\frac{-1}{\sqrt{1-T_{1}^{2}}}\left(1-T_{1}^{2},-T_{1} T_{2},-T_{1} T_{3}\right)
$$

Thus, we have the following theorem. 
Theorem 3.1. Let $T(s), M_{1}(s), M_{2}(s), s \in I$ be three orthonormal unit vectors of the rotation minimizng frame in $\mathbb{E}^{3}$ and let Eq. (2.1) be its derivative formula. Given differentiable functions $k_{1}(s)$ and $k_{2}(s)$, the tangent vector field $T=\left(T_{1}, T_{2}, T_{3}\right)$ in an indirect solution triplet of $E q$. (2.1) is represented in the form

$$
\left\{\begin{array}{l}
T_{1}(s)=\sin f(s) \\
T_{2}(s)=\cos f(s) \cos g(s) \\
T_{3}(s)=\cos f(s) \sin g(s)
\end{array}\right.
$$

such that

$$
\begin{gathered}
f(s)=c_{1}+\int\left(\sin \theta(s) k_{1}(s)-\cos \theta(s) k_{2}(s)\right) d s, \\
g(s)=c_{2}+\int \frac{\left.-\cos \theta(s) k_{1}(s)-\sin \theta(s) k_{2}(s)\right)}{\cos f(s)} d s
\end{gathered}
$$

where $c_{1}$ and $c_{2}$ are constants of integration.

Proof. In this proof, we shall use the base vectors of the new coordinate system. Writing $i=1, j=2$ into Eq.(3.1) and reasoning as $e_{1}^{\prime \prime}=T=\left(T_{1}, T_{2}, T_{3}\right)$, Eqs. (3.2) and (3.3) hold. Also, from Eq. (3.1), a relationship between rotation minimizing vector fields and the vector fields of the new system becomes

$$
\begin{aligned}
& M_{1}(s)=\cos \theta(s) e_{2}^{\prime \prime}(s)-\sin \theta(s) e_{3}{ }^{\prime \prime}(s) \\
& M_{2}(s)=\sin \theta(s) e_{2}{ }^{\prime \prime}(s)+\cos \theta(s) e_{3}^{\prime \prime}(s) .
\end{aligned}
$$

We now calculate the components of the tangent vector field $T(s)$. Writing Eqs. (3.2) and (3.3) into Eq. (3.6) and substituting it into Eq. (2.1), we have

$$
\begin{gathered}
\frac{d T_{1}}{d s}=\left\{\sin \theta(s) k_{1}(s)-\cos \theta(s) k_{2}(s)\right\} \sqrt{1-T_{1}^{2}}, \\
\frac{d T_{2}}{d s}=\frac{1}{\sqrt{1-T_{1}^{2}}}\left[\left\{\cos \theta(s) k_{1}(s)+\sin \theta(s) k_{2}(s)\right\} T_{3}+\left\{\cos \theta(s) k_{2}(s)-\sin \theta(s) k_{1}(s)\right\} T_{1} T_{2}\right], \\
\frac{d T_{3}}{d s}=\frac{-1}{\sqrt{1-T_{1}^{2}}}\left[\left\{\cos \theta(s) k_{1}(s)+\sin \theta(s) k_{2}(s)\right\} T_{2}+\left\{\sin \theta(s) k_{1}(s)-\cos \theta(s) k_{2}(s)\right\} T_{1} T_{3}\right] .
\end{gathered}
$$

Since Eq. (3.7) is a seperable differential equation, it is easier to solve than others and so the solution becomes

$$
T_{1}=\sin \underbrace{\left[c_{1}+\int\left(\sin \theta(s) k_{1}(s)-\cos \theta(s) k_{2}(s)\right) d s\right]}_{=f(s)} .
$$

On the other hand, since Eqs. (3.8) and (3.9) are non-linear differential equations, it is quite advantageous to define a new variable $g(s)$ instead of solving them, satisfying the following statement:

$$
T_{1}^{2}+T_{2}^{2}+T_{3}^{2}=1
$$

from which

$$
T_{2}=\cos f(s) \cos g(s), T_{3}=\cos f(s) \sin g(s) .
$$

Putting Eqs. (3.10) and (3.11) into Eq. (3.8), we have the following variable after a straightforward computations:

$$
g(s)=c_{2}+\int \frac{\left(-\cos \theta(s) k_{1}(s)-\sin \theta(s) k_{2}(s)\right)}{\cos f(s)} d s
$$

which completes the proof. 


\section{Applications}

One of the important areas of study of the differential geometry is the theory of curves that serves a wide range of application areas from biological molecules (DNA, many proteins, etc.) to material science (carbon nano-tube, magnetic field, helical pipes, $\alpha$-helix, etc.). The common property of the curves observed in these areas is that either the vector field or the Darboux vector of the moving frame makes a constant angle with a fixed line in space. For the rotation minimizing frame, there are two curves having the property which refers to above, respectively:

Bishop Slant Helix: A smooth curve is said to be a Bishop slant helix if the vector field $M_{1}$ (or $M_{2}$ ) makes a constant angle with a fixed line in space. Analytically, it characterizes by the constancy of the ratio of the rotation minimizing curvatures $k_{1}$ and $k_{2}$, given by B. Bükcü and M. K. Karacan in the Euclidean 3-space $\mathbb{E}^{3}$ [8].

Darboux Helix: A smooth curve is said to be a Darboux helix if the unit Darboux vector $w_{0}=\frac{w}{\|w\|}=$ $\frac{1}{\sqrt{k_{1}^{2}+k_{2}^{2}}}\left(-k_{2} M_{1}+k_{1} M_{2}\right)$ makes a constant angle with a fixed line in space. The other approach for the Darboux helix is that the spherical indicatrix of the Darboux vector belongs to a circle or a part of circle. With this approach, we see that the curvatures $k_{1}$ and $k_{2}$ of a Darboux helix satisfy the following equation

$$
\frac{\left(k_{1}^{2}+k_{2}^{2}\right)^{\frac{3}{2}}}{k_{1}^{2}} \frac{1}{\left(\frac{k_{2}}{k_{1}}\right)^{1}}=\text { constant }
$$

In Eq. (4.1), remark that the ratio $\frac{k_{2}}{k_{1}}$ must not be constant. According to the theorem (2.1), a Bishop slant helix is a general helix, but not a Darboux helix.

In the previous section, a new coordinate system is introduced in such a way that rotation minimizing frame's derivative formulas are integrable. As a result of this, we obtain a theorem that presents only one component of the indirect solution triplet of Eq. (2.1). In this section, we analyze the necessary condition for the indirect solution to be a steady solution. Interpreted another way, we evaluate what the integration measure is.

For this, we begin with finding the vector fields $M_{1}$ and $M_{2}$ for the tangent vector field $T$ in the Theorem 3.1:

Substituting Eqs. (3.2) and (3.3) into Eq. (3.6), for $M_{1}=\left(M_{1_{1}}, M_{1_{2}}, M_{1_{3}}\right)$ and $M_{2}=\left(M_{2_{1}}, M_{2_{2}}, M_{2_{3}}\right)$, we get

$$
\left\{\begin{array}{l}
M_{1_{1}}=\sin \theta \cos f \\
M_{1_{2}}=\cos \theta \sin g-\sin \theta \sin f \cos g \\
M_{1_{3}}=-\cos \theta \cos g-\sin \theta \sin f \sin g
\end{array}\right.
$$

and

$$
\left\{\begin{array}{l}
M_{2_{1}}=-\cos \theta \cos f \\
M_{2_{2}}=-\sin \theta \sin g+\cos \theta \sin f \cos g \\
M_{2_{3}}=-\sin \theta \cos g+\cos \theta \sin f \sin g
\end{array}\right.
$$

In relation to that, our aim is to find the curvatures $\overline{k_{1}}$ and $\overline{k_{2}}$ of the curve $\bar{r}$, respectively. It is well-known that the curvatures of a curve are invariant up to a rigid motion. For this reason, the curvatures $\overline{k_{1}}$ and $\overline{k_{2}}$ must provide the following equalities:

$$
k_{1}=\overline{k_{1}}, \quad k_{2}=\overline{k_{2}},
$$

where $k_{1}$ and $k_{2}$ are the curvatures of the curve $r$. By differentiating Eq. (4.2) with respect to $s$, we have

$$
\left(M_{1_{1}}^{\prime}\right)^{2}=\cos ^{2} \theta \cos ^{2} f\left(\frac{d \theta}{d s}\right)^{2}+\sin ^{2} \theta \sin ^{2} f\left(\frac{d f}{d s}\right)^{2}-2 \sin \theta \cos \theta \sin f \cos f\left(\frac{d \theta}{d s}\right)\left(\frac{d f}{d s}\right)
$$




$$
\begin{aligned}
& \left(M_{1_{2}}^{\prime}\right)^{2}=\left[\cos ^{2} \theta \sin ^{2} f \cos ^{2} g+2 \cos \theta \sin \theta \sin f \cos g \sin g+\sin ^{2} \theta \sin ^{2} g\right]\left(\frac{d \theta}{d s}\right)^{2} \\
& +\left[\sin ^{2} \theta \sin ^{2} f \sin ^{2} g+2 \cos \theta \sin \theta \sin f \cos g \sin g+\cos ^{2} \theta \cos ^{2} g\right]\left(\frac{d g}{d s}\right)^{2} \\
& +\sin ^{2} \theta \cos ^{2} f \cos ^{2} g\left(\frac{d f}{d s}\right)^{2}+2\left[\sin \theta \cos \theta \sin f \cos f \cos ^{2} g+\sin ^{2} \theta \cos f \sin g \cos g\right]\left(\frac{d \theta}{d s}\right)\left(\frac{d f}{d s}\right) \\
& -2\left[\sin \theta \cos \theta \sin ^{2} f \cos g \sin g+\cos ^{2} \theta \sin f \cos ^{2} g+\sin ^{2} \theta \sin f \sin ^{2} g+\sin \theta \cos \theta \sin g \cos g\right]\left(\frac{d \theta}{d s}\right)\left(\frac{d g}{d s}\right) \\
& -2\left[\sin \theta \cos \theta \cos f \cos ^{2} g+\sin ^{2} \theta \sin f \cos f \sin g \cos g\right]\left(\frac{d f}{d s}\right)\left(\frac{d g}{d s}\right) \\
& \left(M_{1_{3}}^{\prime}\right)^{2}=\left[\cos ^{2} \theta \sin ^{2} f \sin ^{2} g-2 \cos \theta \sin \theta \sin f \cos g \sin g+\sin ^{2} \theta \cos ^{2} g\right]\left(\frac{d \theta}{d s}\right)^{2} \\
& +\left[\sin ^{2} \theta \sin ^{2} f \cos ^{2} g-2 \cos \theta \sin \theta \sin f \cos g \sin g+\cos ^{2} \theta \sin ^{2} g\right]\left(\frac{d g}{d s}\right)^{2} \\
& +\sin ^{2} \theta \cos ^{2} f \sin ^{2} g\left(\frac{d f}{d s}\right)^{2}+2\left[\sin \theta \cos \theta \sin f \cos f \sin ^{2} g-\sin ^{2} \theta \cos f \sin g \cos g\right]\left(\frac{d \theta}{d s}\right)\left(\frac{d f}{d s}\right) \\
& +2\left[\sin \theta \cos \theta \sin ^{2} f \cos g \sin g-\cos ^{2} \theta \sin f \sin ^{2} g-\sin ^{2} \theta \sin f \cos ^{2} g+\sin \theta \cos \theta \sin g \cos g\right]\left(\frac{d \theta}{d s}\right)\left(\frac{d g}{d s}\right) \\
& +2\left[-\sin \theta \cos \theta \cos f \sin ^{2} g+\sin ^{2} \theta \sin f \cos f \sin g \cos g\right]\left(\frac{d f}{d s}\right)\left(\frac{d g}{d s}\right),
\end{aligned}
$$

from which

$$
\begin{aligned}
& M_{1_{1}}^{\prime}=\cos \theta \cos f \frac{d \theta}{d s}-\sin \theta \sin f \frac{d f}{d s} \\
& M_{1_{2}}^{\prime}=\{-\cos \theta \sin f \cos g-\sin \theta \sin g\} \frac{d \theta}{d s}-\sin \theta \cos f \cos g \frac{d f}{d s}+\{\sin \theta \sin f \sin g+\cos \theta \cos g\} \frac{d g}{d s} \\
& M_{1_{3}}^{\prime}=\{-\cos \theta \sin f \sin g+\sin \theta \cos g\} \frac{d \theta}{d s}-\sin \theta \cos f \sin g \frac{d f}{d s}-\{\sin \theta \sin f \cos g-\cos \theta \sin g\} \frac{d g}{d s} .
\end{aligned}
$$

From the above relations, we find

$$
\overline{k_{1}}=\sqrt{\left(\frac{d \theta}{d s}+\sin f \frac{d g}{d s}\right)^{2}+k_{1}^{2}} .
$$

In a similar way, it is easy to see that another curvature is given by

$$
\overline{k_{2}}=\sqrt{\left(\frac{d \theta}{d s}+\sin f \frac{d g}{d s}\right)^{2}+k_{2}^{2}} .
$$

By this way, we have the following lemma that states a criteria for the integration measure.

Lemma 4.1. Let $r(s)$ be a curve parametrized by the arc-length $s$ in the Euclidean 3-space $\mathbb{E}^{3}$ and the differentiable functions $k_{1}(s)$ and $k_{2}(s)$ be the rotation minimizing curvatures of $r$. If the following relation holds

$$
\frac{d \theta}{d s}+\sin f(s) \frac{d g}{d s}=0
$$

then there exist "steady" solutions satisfying Eq. (2.1), where $f(s)$ and $g(s)$ are given by Eqs. (3.4) and (3.5), respectively. 
From Lemma 4.1, we can consider two cases as follows:

Case 1 When $\theta=$ constant, Eq. (4.4) is reduced to

$$
\sin f(s) \frac{d g}{d s}=0
$$

Subcase $1.1(\sin f(s)=0)$. This means that $f=0$ or $f=2 \pi k, k \in \mathbb{Z}$. From this fact, it is possible to regard the integrand in Eq. (3.4) as

$$
\sin \theta k_{1}(s)-\cos \theta k_{2}(s)=0
$$

The last equality implies that the following relations hold:

- If $\sin \theta=0$, then $k_{2}=0$, which is a contradiction.

- If $\cos \theta=0$, then $k_{1}=0$, which is a contradiction.

- If $\sin \theta \neq 0$ and $\cos \theta \neq 0$, the desirable curve is a Bishop slant helix with

$$
\frac{k_{2}}{k_{1}}=\tan \theta_{0}=m \neq 0,
$$

where $\theta_{0}$ is determined by the curvatures.

Observe that the other function $g(s)$ can be found according to the above relation using Eq.(4.5) such that

$$
g(s)=c_{2}+\frac{m}{n} \int k_{1}(s) d s
$$

where $m=\frac{n}{\sqrt{1-n^{2}}}$. Also, we can find its straight line $d=(a, b, c)$ using Eq. (4.2):

$$
g\left(M_{1}, d\right)=\frac{m}{\sqrt{1+m^{2}}} a+\frac{1}{\sqrt{1+m^{2}}}\{b \sin g(s)-c \cos g(s)\} .
$$

According to the definition of Bishop slant helices, the above dot product is constant if and only if the following relations hold:

$$
\begin{gathered}
b \sin g(s)-c \cos g(s)=0, \\
a= \pm 1,
\end{gathered}
$$

from which, we get $d=( \pm 1,0,0)$. This gives information about the plane in which the straight line is spanned. Note that $g\left(M_{1}, d\right)=n$.

Subcase $1.2\left(\frac{d g}{d s}=0\right)$. This means that $g=$ constant. From this fact, it is possible to regard the integrand in Eq. (3.5) as

$$
-\cos \theta k_{1}(s)-\sin \theta k_{2}(s)=0 .
$$

From the last equality, we have the following relations:

- If $\cos \theta=0$, then $k_{2}=0$, which is a contradiction.

- If $\sin \theta=0$, then $k_{1}=0$, which is a contradiction.

- If $\cos \theta \neq 0$ and $\sin \theta \neq 0$, the desirable curve is a Bishop slant helix with

$$
\frac{k_{2}}{k_{1}}=-\cot \theta_{1}=m
$$

where $\theta_{1}=\frac{\pi}{2}+\theta_{0}$.

Observe that the other function $f(s)$ can be found according to the above relation using Eq.(4.7) such that

$$
f(s)=c_{1}+\frac{m}{n} \int k_{1}(s) d s
$$


where $m=\frac{n}{\sqrt{1-n^{2}}}$. Also, we can find its straight line $d=(a, b, c)$ using Eq. (4.2):

$$
g\left(M_{1}, d\right)=\frac{-1}{\sqrt{1+m^{2}}} \sin f\{b \cos C+c \sin C\}+\frac{a}{\sqrt{1+m^{2}}} \cos f+\frac{m}{\sqrt{1+m^{2}}}\{b \sin C-c \cos C\},
$$

where $C$ is determined by the constancy of $g$. According to the definition of a Bishop slant helix, the above dot product is constant if and only if the following relations hold:

$$
\begin{gathered}
b \cos C+c \sin C=0, \\
b \sin C-c \cos C=1, \\
a=0,
\end{gathered}
$$

from which, we get $d=(0,-\sin C, \cos C)$. This gives information about the plane in which the straight line is spanned. Note that $g\left(M_{1}, d\right)=n$.

Now, together with the subcases (1.1) and (1.2), putting Eqs. (4.5-4.6) and (4.7-4.8) into the Theorem 3.1, we have the following propositions that present the natural representations of the position vector field of a Bishop slant helix with $S p\left\{e_{1}\right\}$ and $S p\left\{e_{2}, e_{3}\right\}$, respectively:

Proposition 4.1. Let $r$ be a Bishop slant helix in $\mathbb{E}^{3}$ with curvature function $k_{1}$. Thus, its position vector field is computed in the natural representation form as follows:

$$
r(s)=\left(d_{1}, \int \cos \left[c_{2}+\frac{m}{n} \int k_{1}(s) d s\right] d s+d_{2}, \int \sin \left[c_{2}+\frac{m}{n} \int k_{1}(s) d s\right] d s+d_{3}\right)
$$

where $c_{2}$ and $d_{i}$ for $i=1,2,3$ are constants of integration, $m=\frac{n}{\sqrt{1-n^{2}}}, n=\sin \left[\theta_{0}\right]$ and $\theta_{0}$ is the angle between the fixed straight line $e_{1}$ (axis of a Bishop slant helix) and the vector $M_{1}$ of the curve $r$.

Proposition 4.2. Let $r$ be a Bishop slant helix in $\mathbb{E}^{3}$ with curvature function $k_{1}$. Thus, its position vector field is computed in the natural representation form as follows:

$$
\begin{aligned}
r(s)=\left(\int \sin \left[c_{1}+\frac{m}{n} \int k_{1}(s) d s\right] d s+d_{1}, \cos C \int \cos \left[c_{1}+\frac{m}{n} \int k_{1}(s) d s\right] d s+d_{2},\right. \\
\left.\quad \sin C \int \cos \left[c_{1}+\frac{m}{n} \int k_{1}(s) d s\right] d s+d_{3}\right),
\end{aligned}
$$

where $C, c_{1}$ and $d_{i}$ for $i=1,2,3$ are constants of integration, $m=\frac{n}{\sqrt{1-n^{2}}}, n=\sin \left[\theta_{0}\right]$ and $\theta_{0}$ is the angle between the fixed straight line $d=\left(0,-\sin C, \cos C\right.$ ) (axis of a Bishop slant helix) and the vector field $M_{1}$ of the curve $r$.

Corollary 4.1. A Bishop slant helix with the rotation minimizing curvature functions $k_{1}(s)$ and $k_{2}(s)$ in $\mathbb{E}^{3}$ is a plane curve having an analytical property such that the ratio of $k_{1}(s)$ to the Frenet curvature $\kappa(s)$ is constant.

Example 4.1. As an application of the Proposition 4.1., we compute the RMF vector fields of a Bishop slant helix with $d \in S p\left\{e_{1}\right\}$ for $k_{1}(s)=s$ and $m=\frac{1}{3}$ as follows: From Theorem 3.1, we can write

$$
T(s)=\left(0, \cos \left(\frac{\sqrt{10}}{3} s^{2}\right), \sin \left(\frac{\sqrt{10}}{3} s^{2}\right)\right) .
$$

From Eqs. (4.2) and (4.3), we have

$$
M_{1}(s)=\frac{1}{\sqrt{10}}\left(1,3 \sin \left(\frac{\sqrt{10}}{3} s^{2}\right), 3 \cos \left(\frac{\sqrt{10}}{3} s^{2}\right)\right)
$$

and

$$
M_{2}(s)=\frac{1}{\sqrt{10}}\left(-3, \sin \left(\frac{\sqrt{10}}{3} s^{2}\right), \cos \left(\frac{\sqrt{10}}{3} s^{2}\right)\right) .
$$

Observe that $g\left(M_{1}, e_{1}\right)=\frac{m}{\sqrt{1+m^{2}}}$. Also, by a long computation, we see that $\kappa(s)=\frac{2 \sqrt{10}}{3} s$ and $\tau(s)=0$, which supports the Corollary 4.1.

We now plot it putting $d_{1}=d_{2}=d_{3}=c_{2}=0$ with respect to the axis of the Bishop slant helix. 

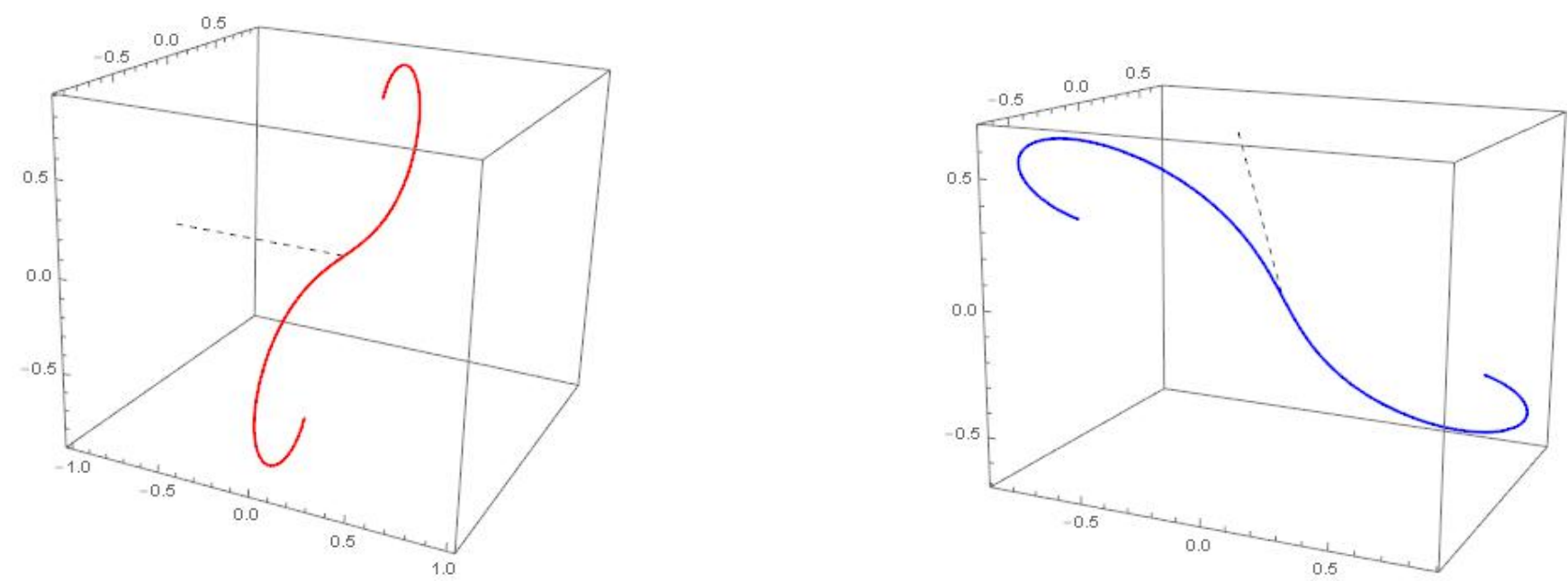

Figure 3. The graphic with the color red is $d \in S p\left\{e_{1}\right\}$ and the graphic with the color blue is $d \in S p\left\{e_{2}, e_{3}\right\}$. The dashed lines represent the axis of the Bishop slant helices.

We now can consider the last case.

Case 2 When $\theta \neq$ constant, we have three subcases as follows:

- $f=$ constant and $g=$ constant

- $f \neq$ constant and $g=$ constant

- $f=$ constant and $g \neq$ constant.

Considering the first two items, we get a contradiction about the fact that $\theta \neq$ constant. Thus, this subcases never occur. We now can consider the last item as follows:

The constancy of the function $f$ implies that

$$
\sin \theta(s) k_{1}(s)-\cos \theta(s) k_{2}(s)=0 .
$$

Substituting the last relation into Eq. (3.5), we find the other function $g$ as

$$
c_{2}+\frac{-1}{n} \int \sqrt{k_{1}^{2}(s)+k_{2}^{2}(s)} d s,
$$

where $n=\cos c_{1}$. Thus, Eq. (4.4) is expressed by

$$
\frac{d \theta}{d s}-m \sqrt{k_{1}^{2}(s)+k_{2}^{2}(s)}=0,
$$

from which, we get

$$
\theta(s)=m \int \sqrt{k_{1}^{2}(s)+k_{2}^{2}(s)} d s
$$

where $m=\frac{\sqrt{1-n^{2}}}{n}$. From Eqs. (4.9) and (4.11), we obtain

$$
m=\frac{k_{1}^{2}\left(\frac{k_{2}}{k_{1}}\right)^{\prime}}{\left(k_{1}^{2}+k_{2}^{2}\right)^{\frac{3}{2}}},
$$

where since the ratio of $k_{2}(s)$ to $k_{1}(s)$ is non-constant due to Eq. (4.9), $m \neq 0$. This means that Eq. (4.12) can be reversible relative to multiplication, that is

$$
\frac{1}{m}=\frac{\left(k_{1}^{2}+k_{2}^{2}\right)^{\frac{3}{2}}}{k_{1}^{2}} \frac{1}{\left(\frac{k_{2}}{k_{1}}\right)^{1}}=\text { constant } .
$$


Also, we can find its straight line $d=(a, b, c)$ :

$$
g\left(w_{0}, d\right)=-\frac{m}{\sqrt{1+m^{2}}} a+\frac{1}{\sqrt{1+m^{2}}}\{b \sin g(s)-c \cos g(s)\} .
$$

According to the definition of Darboux helices, the above dot product is constant if and only if the following relations hold:

$$
\begin{gathered}
b \sin g(s)-c \cos g(s)=0, \\
a= \pm 1,
\end{gathered}
$$

from which, we get $d=( \pm 1,0,0)$. This gives information about the plane in which the straight line is spanned. Note that $g\left(w_{0}, d\right)=n$. By way of Eq. (4.1), putting Eqs. (4.9) and (4.10) into the theorem 3.1, we have a natural representation of the position vector of a Darboux helix.

Proposition 4.3. Let $r$ be a Darboux helix in $\mathbb{E}^{3}$ and $k_{1}(s), k_{2}(s)$ be the rotation minimizing curvature functions of the curve. Thus, its position vector field is computed in the natural representation form as follows:

$$
\begin{aligned}
& r(s)=n\left(m s+d_{1}, \int \cos \left(c_{2}+\sqrt{1+m^{2}} \int \sqrt{k_{1}^{2}(s)+k_{2}^{2}(s)} d s\right) d s+d_{2},\right. \\
& \left.\qquad \sin \left(c_{2}+\sqrt{1+m^{2}} \int \sqrt{k_{1}^{2}(s)+k_{2}^{2}(s)} d s\right) d s+d_{3}\right),
\end{aligned}
$$

where $c_{2}$ and $d_{i}$ for $i=1,2,3$ are constants of integration, $0 \neq m=\frac{\sqrt{1-n^{2}}}{n}, n=\cos [\phi]$ and $\phi$ is the angle between the fixed straight line $e_{1}$ (axis of a Darboux helix) and the unit Darboux vector $w_{0}$ of the curve $r$.

Remark 4.1. Writing 1 instead of $n$ in the above proposition, one see that the position vector of the Darboux helix become that of the Bishop slant helix. By this way, we have the following corollary.

Corollary 4.2. General helices consist of slant helices and Darboux helices in the sense of Bishop.

Example 4.2. As an application of the proposition 4.3, we see that a natural representation of the position vector of the curve having the curvatures $k_{1}(s)=\tan (\arcsin m s)$ and $k_{2}(s)=1$ from the family of Darboux helices is as follows.

$$
\begin{aligned}
r(s)=\left(\frac{m}{\sqrt{1+m^{2}}} s+d_{1}, \frac{1}{\sqrt{1+m^{2}}} \int \cos \left[c_{2}+\frac{-\sqrt{1+m^{2}}}{m} \arcsin m s\right] d s+d_{2},\right. \\
\left.\frac{1}{\sqrt{1+m^{2}}} \int \sin \left[c_{2}+\frac{-\sqrt{1+m^{2}}}{m} \arcsin m s\right] d s+d_{3}\right),
\end{aligned}
$$

where since the curvature $k_{1}$ is non-zero, $m \neq 0$. Also, by a long computation, the torsion of $r$ equals to $\frac{-m}{\sqrt{1-(m s)^{2}}}$. This implies that the smooth curve $r$ is a general helix, but not Bishop slant helix, which supports the corollary 4.2. Observe that the curve $r$ is a Bishop slant helix if $m=0$. We now can plot it putting $d_{1}=d_{2}=d_{3}=c_{2}=0$ with respect to the specific values of $m$.

Remark 4.2. In this work, we analyze the results when only $i=1$ in order to avoid repetition. The geometric meaning of results in the case where $i=2$ (or $i=3$ ) is the displacement of the components of the curve.

\section{Conclusion}

The scope of the paper covers two different problems on the same baseline. The first one is to introduce a new method in such a way that the derivative formulas of the rotation minimizing frame in the Euclidean space can easily be integrated. The base of this method consists of the new basis vectors resulting in the rotations. In the formula containing these basis vectors, we choose $i=1$. In that case, one can say that the tangent vector field $T$ might be parallel to the vector field $e_{1}$. However, it should be remarked that we have not established an alternative frame on the curve, which is defined at every point. Indeed, we establish a new system by means of a rigid motion such that the vector field $T$ can coincide with the vector field $e_{1}^{\prime \prime}$ for every appropriate points on the curve that do not satisfy $e_{1} / / T$. The second one is to compute the position vector field of a smooth curve having a linear relationship between the rotation minimizing curvatures, which couldn't be obtained by the present methods. Thus, we brought a new approach to the open problem which we referred to the prior and we obtained the position vector field of slant helices and Darboux helices in the sense of Bishop. 

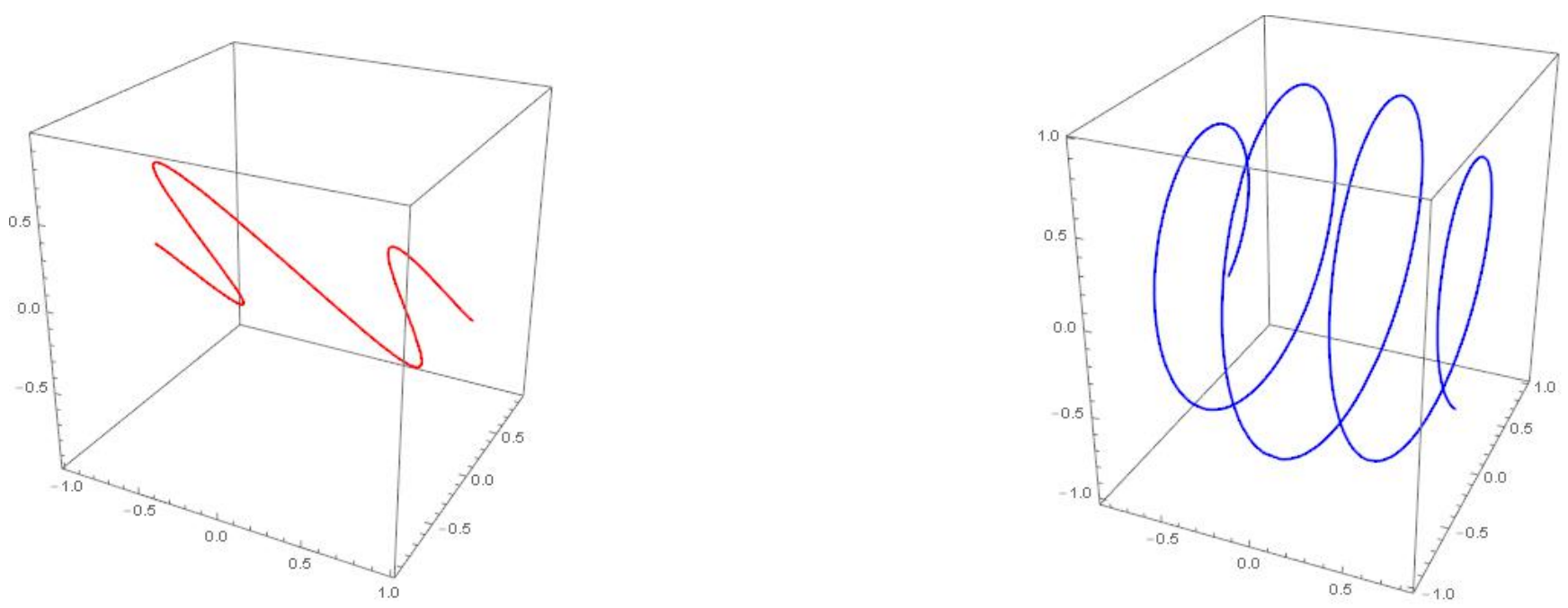

Figure 4. Some Darboux helices with $k_{1}(s)=\tan (\arcsin m s), k_{2}(s)=1$ for $m=\frac{1}{5}$ (red) and $m=\frac{1}{12}$ (blue).

\section{References}

[1] Ali, A.T., Turgut, M.: Position vector of a time-like slant helix in Minkowski 3-space. J. Math. Analysis and Appl. 365(2), 559-569 (2010).

[2] Ali, A.T.: Position vectors of slant helices in Euclidean 3-space. J. Egyptian Math. Soc. 20(1), 1-6 (2012).

[3] Ali, A.T.: Position vectors of general helices in Euclidean 3-space. Bull. Math. Anal. Appl. 3(2), 198-205 (2010).

[4] Ali, A.T., Turgut, M.: Position vectors of timelike general helices in Minkowski 3-space. Glo. J. Adv. Res. Class. Mod. Geom. 2(1), 2-10 (2013).

[5] Ali, A.T.: Position vectors of spacelike general helices in Minkowski 3-space. Nonlinear Analysis: Theory, Methods and Applications. 73(4), 1118-1126 (2010).

[6] Ali, A.T.: Position vectors of curves in the Galilean space $\mathbb{G}_{3}$. Matematicki Vesnik. 64(3), 200-210 (2012).

[7] Bishop, R.L.: There is more than one way to frame a curve. The American Mathematical Monthly. 82(3), $246-251$ (1975).

[8] Bükcü B., Karacan, M.K.: The slant helices according to Bishop frame. I. J. Computational and Math. Sci. 3(2), 67-70 (2009).

[9] Carmo, M.D.: Differential Geometry of Curves and Surfaces. Prentice-Hall. New Jersey (1976).

[10] Choi, J.H., Kim, Y.H.: Associated curves of a frenet curve and their applications. Applied Mathematics and Computation, 218(18), 9116-9124 (2012).

[11] Kizıltuğ, S., Önder, M.: Associated curves of frenet curves in three dimensional compact lie group. Miskolc Mathematical Notes. 16(2), 953-694 (2015).

[12] Kim, Y.H., Choi J.H., Ali, A.T.: Some associated curves of frenet non-lightlike curves in $\mathbb{E}_{1}^{3}$. J. Math. Analy. Appl. 394(2), 712-723 (2012).

[13] Lucas, P., Ortega-Yagues, J.A.: Slant helices in the euclidean 3-space revisited. Bull. Belgian Math. Soc. Simon Stevin. 23(1), 133-150 (2016).

[14] Macit, N., Akbiyık, M., Yüce, S.: Some new associated curves of an admissible frenet curve in 3-dimensional and 4-dimensional Galilean spaces. Romanian J. Math. Computer Sci. 7(2), 110-122 (2017).

[15] Mak, M., Altınbaş, H.: Some special associated curves of non-degenerate curve in anti de sitter 3-space. Math. Sci. Appl. E-Notes. 5(2), 89-97 (2017).

[16] Öztekin, H., Tatlipınar, S.: Determination of the position vectors of curves from intrinsic equations in $\mathbb{G}_{3}$. Walailak J. Sci. Tech. 11(12), 1011-1018 (2014).

[17] Reich, K.: Die geschichte der differential geometrie von gauss bis Riemann. Archive for History of Exact Sciences. 11(4), 273-376 (1973).

[18] Savc1, U.Z., Y1lmaz, S., Mağden, A.: Position vector of some special curves in Galilean 3-spaces $\mathbb{G}_{3}$. Glo. J. Adv. Res. Class. Mod. Geom. 3(1), 7-11 (2014).

\section{Affiliations}

\section{FIRAT YERLIKAYA}

ADDRESS: Ondokuz Mayis University, Dept. of Mathematics, 55139, Samsun-Turkey.

E-MAIL: firat.yerlikaya@omu.edu.tr

ORCID ID: 0000-0003-2360-1522

\section{İSMAİL AYDEMİR}

AdDRESS: Ondokuz Mayis University, Dept. of Mathematics, 55139, Samsun-Turkey.

E-MAIL: iaydemir@omu.edu.tr

ORCID ID: 0000-0002-0238-2079 